\title{
Axon Diameter Mapping in the Presence of Orientation Dispersion with Diffusion MRI
}

\author{
Hui Zhang and Daniel C. Alexander \\ Microstructure Imaging Group, Department of Computer Science, University College \\ London, London WC1E 6BT, United Kingdom
}

\begin{abstract}
Direct measurement of tissue microstructure with diffusion MRI offers a new class of biomarkers, such as axon diameters, that give more specific information about tissue than measures derived from diffusion tensors. The existing techniques of this kind assume a single axon orientation in the tissue model, which may be a reasonable approximation only for the most coherent brain white matter, such as the corpus callosum. For most other areas, orientation dispersion is not negligible and, if unaccounted for, leads to overestimation of the axon diameters, prohibiting their accurate mapping over the whole brain. Here we propose a new model that captures the effect of orientation dispersion explicitly. An efficient numerical scheme is developed to enable the axon diameter estimation by fitting the proposed model. Synthetic data experiments demonstrate that the new model provides an axon diameter index that is robust to the presence of orientation dispersion. Results on in vivo human data show reduced axon diameter index and better agreement with histology compared to previous methods suggesting improvements in the axon diameter estimate.
\end{abstract}

\section{Introduction}

Diffusion MRI reveals tissue microstructure by measuring patterns of water diffusion as influenced by the microscopic environment of the tissue. The most popular technique of this kind is the diffusion-tensor imaging (DTI) [1. It provides simple biomarkers, such as mean diffusivity (MD) and fractional anisotropy (FA), that are effective at indicating major microstructural changes in tissue during normal development or due to disease. However, a limitation of these biomarkers is that they are inherently non-specific [2], e.g. changes in FA may be attributed to changes in a combination of the underlying microstructure features, such as axon density and diameter, and cannot isolate their individual contributions. Direct measurement of these features can shed new light into development and disease mechanisms by offering a new class of highly-specific biomarkers.

Previous work, e.g. [3, 4,5,6,7, shows the feasibility of using diffusion MRI to measure microstructure features directly in excised biological tissues as well as in vivo. A particularly successful approach, exemplified by [3,4], is the model-based strategy in which a geometric model of the microstructure of interest predicts the MR signal from water diffusing within. In estimating axon diameters using this 
approach, the earlier attempts, e.g. [5,7], assume a single and known orientation of axon in the tissue model. Alexander [6] demonstrates in simulation that it is possible to estimate axon diameters of unknown orientation using a multi-shell high angular resolution diffusion-weighted imaging (HARDI) protocol optimized with realistic in vivo diffusivities and constraints typical of a clinical scanner. Later work [8] gives promising results in both fixed monkey brains and live human brains that show trends in the midsagittal corpus callosum (CC) and cortico-spinal tract (CST) that we expect from histology.

The model in [8] simplifies real white matter (WM) in two important ways: it assumes a single axon diameter rather than a distribution; it assumes all the axons within single voxels have the same single orientation. The first simplification means that the fitted model provides a single index (summary statistic) of the axon diameter distribution rather than the full distribution. The index correlates with the mean diameter weighted by volume so is still useful. The second simplification holds for all previous methods of axon diameter estimation [3, 4, 5, 7] and limits estimates only to the most coherent structures, e.g. CC; even there the assumption of coherent orientation is questionable. Most brain WM has significant orientation dispersion. This leads to the overestimation of the axon diameter index or distribution because axons oblique to the assumed single orientation appear to have larger cross section, as shown in Fig. 2(a). The limitation prevents the accurate mapping of their microstructure features over the whole brain and casts doubt on estimates even from CC and CST.

Here we aim to ameliorate this limitation by introducing a new tissue model that explicitly represents the dispersion in axon orientation. An efficient numerical scheme makes the proposed model feasible for axon diameter estimation. Experimental results demonstrate that the proposed model estimates axon diameter indices from synthetic data and human brain data, and other microstructure features, more accurately than previous ones. The rest of the paper is organized as follows: Section 2 describes the proposed tissue model and its numerical implementation; Section 3 gives the experimental design and results; Section 4 summarizes the contribution and discusses future work.

\section{Tissue and Signal Model}

The proposed model is based on the simplified version [6] of Assaf et al.'s composite hindered and restricted model of diffusion [4. It models WM as a population of cylindrical axons with a single radius and impermeable cell walls embedded in a homogeneous medium as in $[3,4,9,5,6,7,7$. To account for the CSF contribution through the partial volume effect, an isotropic compartment is included as in [7]. The normalized MR signal $A(\boldsymbol{G}, \Delta, \delta)$ from the standard pulsed-gradient spinecho (PGSE) sequence, with gradient pulses of length $\delta$, strength and direction $\boldsymbol{G}$, and separation $\Delta$, can be written as

$$
\left(1-\nu_{i s o}\right)\left(\nu_{i c} A_{i c}(\boldsymbol{G}, \Delta, \delta)+\left(1-\nu_{i c}\right) A_{e c}(\boldsymbol{G}, \Delta, \delta)\right)+\nu_{i s o} A_{i s o}(\boldsymbol{G}, \Delta, \delta),
$$

where $\nu_{\text {iso }} \in[0,1]$ is the volume fraction of the isotropic compartment, $\nu_{i c} \in[0,1]$ the relative volume fraction of the intra-cellular compartment among WM, and 
$A_{i c}, A_{e c}$, and $A_{i s o}$ the normalized signals from the intra-cellular, extra-cellular and isotropic compartments respectively.

Intra-Cellular Model. Let the distribution of axon orientation be $\rho: \mathbb{S}^{2} \mapsto$ $\mathbb{R}^{+}$, where $\rho(\boldsymbol{n})=\rho(-\boldsymbol{n})$ and $\int \rho(\boldsymbol{n}) d \boldsymbol{n}=1$. The intra-cellular signal is then

$$
A_{i c}(\boldsymbol{G}, \Delta, \delta \mid \rho, R)=\int \rho(\boldsymbol{n}) A_{c y l}(\boldsymbol{G}, \Delta, \delta \mid \boldsymbol{n}, R) d \boldsymbol{n},
$$

where $A_{c y l}$ is the signal from water restricted by a cylinder of radius $R$ and orientation $\boldsymbol{n}$ for which we use the Gaussian phase distribution approximation [10], as in 6 .

Here we consider two models for $\rho$ :

- The Delta model where $\rho(\boldsymbol{n})$ is a $\delta$-function, which reduces (2) to the form of previous models in [4, 6.

- The Watson model where we model $\rho$ with a Watson distribution [11] so that

$$
\rho(\boldsymbol{n})=f(\boldsymbol{n} \mid \boldsymbol{\mu}, \kappa)=M\left(\frac{1}{2}, \frac{3}{2}, \kappa\right)^{-1} e^{\kappa(\boldsymbol{\mu} \cdot \boldsymbol{n})^{2}},
$$

where $M$ is a confluent hypergeometric function, $\boldsymbol{\mu}$ is the unit vector about which the distribution is cylindrically symmetric, and $\kappa$, the concentration parameter, controls the extent of orientation dispersion.

The Watson distribution is the spherical analog of the Gaussian distribution with cylindrical symmetry. It allows us to capture the essential characteristics of nonparallel axons with $\kappa$, the single extra parameter compared to the Delta model. Although the general admissible values for $\kappa$ range from $-\infty$ to $+\infty$, here we constrain it to be strictly positive such that $\boldsymbol{\mu}$ represents the mean orientation of the distribution with larger $\kappa$ corresponding to lower dispersion about $\boldsymbol{\mu}$.

Extra-Cellular Model. This compartment exhibits hindered diffusion and is modeled with simple (Gaussian) anisotropic diffusion, such that $A_{e c}(\boldsymbol{G}, \Delta, \delta)=$ $e^{-(\Delta-\delta / 3) \gamma^{2} \delta^{2} \boldsymbol{G}^{\mathrm{T}} \mathbb{D}_{e c} \boldsymbol{G}}$, where $\mathbb{D}_{e c}$ is the (apparent) diffusion tensor of the compartment. We define $\mathbb{D}_{e c}$ in terms of the distribution of axon orientation $\rho$ as

$$
\mathbb{D}_{e c}=\int \rho(\boldsymbol{n}) \mathbb{D}_{h}(\boldsymbol{n}) d \boldsymbol{n},
$$

where $\mathbb{D}_{h}(\boldsymbol{n})$ is the diffusion tensor for axons with a single orientation $\boldsymbol{n}$ for which we use the definition $\mathbb{D}_{h}(\boldsymbol{n})=\left(d-d_{\perp}\right) \boldsymbol{n} \boldsymbol{n}^{\mathrm{T}}+d_{\perp} \mathbb{I}$, with $d_{\perp}$ being the apparent diffusion coefficient perpendicular to axons and $\mathbb{I}$ being the identity tensor, as in [4]. For the Delta model, $\mathbb{D}_{e c}=\mathbb{D}_{h}$. For the Watson model, $\mathbb{D}_{e c}=$ $\left(d^{\prime}-d_{\perp}^{\prime}\right) \boldsymbol{\mu} \boldsymbol{\mu}^{\mathrm{T}}+d_{\perp}^{\prime} \mathbb{I}$, where

$$
\begin{aligned}
& d^{\prime}=d+\left(d-d_{\perp}\right)\left(\frac{1}{2 \sqrt{\kappa} \operatorname{DawsonF}(\sqrt{\kappa})}-\frac{1}{2 \kappa}-1\right) \\
& d_{\perp}^{\prime}=d_{\perp}+\left(d-d_{\perp}\right)\left(\frac{1}{4 \kappa}-\frac{1}{4 \sqrt{\kappa} \operatorname{DawsonF}(\sqrt{\kappa})}+\frac{1}{2}\right)
\end{aligned}
$$

and DawsonF is the Dawson's integral [12. We use Szafer et al.'s tortuosity model [13] and set $d_{\perp}=d\left(1-\nu_{i c}\right)$. 
Isotropic Model. The isotropic compartment does not depend on the distribution of axon orientation and is modeled as a simple isotropic diffusion process such that $A_{\text {iso }}=e^{-(\Delta-\delta / 3) \gamma^{2} \delta^{2}\|\boldsymbol{G}\|^{2} D}$ with $D$ being the intrinsic diffusion coefficient of the compartment.

Numerical Implementation. The integral in (2) does not have a closedform analytic solution while brute-force numerical integration is prohibitively expensive, up to 1000 times longer than computing the Delta model. To make parameter estimation using the Watson model feasible, we express the Watson distribution function by its spherical harmonic $(\mathrm{SH})$ expansion which makes the integral in (2) analytic leading to the following summation:

$$
A_{i c}=\sum_{l=0}^{\infty} f_{l 0}(\kappa) \sqrt{\frac{2 l+1}{4 \pi}} P_{l}(\boldsymbol{q} \cdot \boldsymbol{\mu}) G_{l}\left(d, \nu_{i c}, R\right),
$$

where $f_{l 0}(\kappa)$ is the spherical harmonic (SH) coefficient (order $l$ and 0 ) of the canonical Watson distribution $f(\boldsymbol{n} \mid \boldsymbol{\mu}=\boldsymbol{z}, \kappa)$ and has a closed-form analytic expression for all $l, P_{l}$ is the Legendre polynomial of order $l, G_{l}$ is a real function, nonzero only for even $l$, and can be calculated analytically. In the current implementation, the summation is truncated at $l=12$ to give a good approximation for $\kappa$ up to 128. The implementation is orders of magnitude more efficient than numerical integration, taking approximately only $50 \%$ longer than computing the Delta model.

\section{$3 \quad$ Experiments and Results}

This section describes the synthetic and in vivo human data experiments and results that compare the proposed Watson model to the Delta model in terms of their ability to estimate axon diameters and other microstructure parameters. The synthetic experiments compare the accuracy with which known tissue parameters can be recovered by each model while the in vivo experiments compare each model's performance in real human data.

Data Acquisition. In vivo imaging uses a clinical 3T Philips scanner to acquire 360 diffusion-weighted images of a healthy volunteer in about an hour with the protocol in [8]. The protocol, determined via the optimization procedure in [6], divides the measurements into 4 HARDI shells each with 90 gradient directions and includes $4 b=0$ images. It uses sagittal echo-planar imaging with in-plane resolution $128 \times 128$ with $1.8 \times 1.8 \mathrm{~mm}^{2}$ voxels and thickness $3.9 \mathrm{~mm}$, no gap, resulting in white matter signal-to-noise (SNR) at $b=0$ about 20 .

Model Fitting. For both experiments, we fit the models to data with the Rician Markov Chain Monte Carlo (MCMC) procedure in [6], after an initial grid search and gradient descent to determine the maximum likelihood (ML) estimates of the parameters. The MCMC procedure refines the ML estimates for $R$ and $\nu_{i c}$, for both models, and $\kappa$, for the Watson model, by computing their 

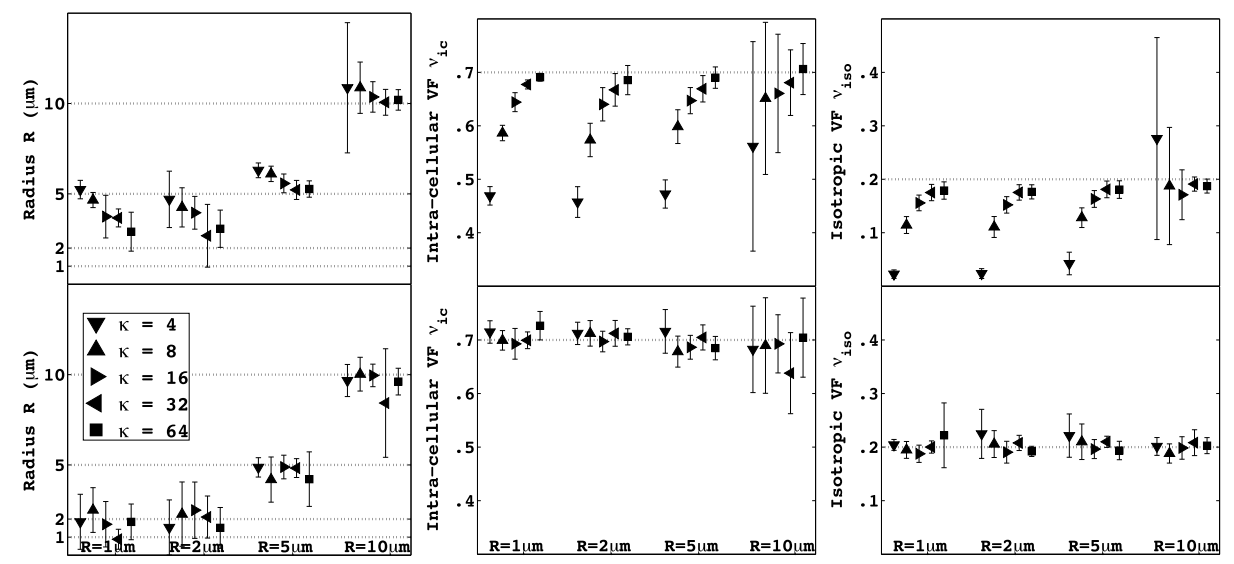

Fig. 1. The parameter estimates from the synthetic experiments using the Delta (top) and Watson (bottom) models. The dotted lines indicate the true parameter values.

posterior distributions, the means of which provide the final estimates for these parameters. Throughout, we fix $d$ and $D$ to their expected values in human in vivo data, which are 1.7 and $3.0 \times 10^{-9} \mathrm{~m}^{2} \mathrm{~s}^{-1}$, respectively. The MCMC setting is 40 samples at intervals of 200 iterations after a burn in of 2000 iterations.

Synthetic Experiments. We synthesize MR data from the proposed Watson model using the imaging protocol of the human data described above and adds synthetic Rician noise with $\sigma=0.05$ to match the SNR of the human data. The true model parameters are $\nu_{i c}=0.7, \nu_{i s o}=0.2, R \in\{1,2,5,10\} \mu \mathrm{m}$, and $\kappa \in\{4,8,16,32,64\}$. We test 10 different orientations, uniformly distributed over the sphere. For each parameter, we report the mean and standard deviation of its 10 estimates over the different orientations.

Fig. 1 shows the recovered model parameters for both the Delta and Watson models. The Delta model consistently overestimates the axon radius, as expected, with the effect more pronounced for small radii and large orientation dispersion (i.e., lower $\kappa$ ). It also consistently underestimates $\nu_{i c}$ with increasing bias for decreasing $\kappa$. The estimate for $\nu_{i s o}$ is similarly biased downward except for the largest value of $R$ which is biased upward. For the very high $\kappa$ values, 32 and 64 , the bias in the estimation becomes negligible for all the parameters.

In contrast, the Watson model provides consistently more accurate estimates for these parameters with just a few exceptions. It overestimates the smallest value of $R$, making it indistinguishable from $R=2 \mu \mathrm{m}$, but the overestimation is slight, which does not prevent it from being distinguished from $R=5 \mu \mathrm{m}$. The model slightly underestimates the radius for some $\kappa$ and $R$ values but there is no systematic pattern.

The Watson model also supports the direct estimation of orientation dispersion via $\kappa$, the results of which are shown in Fig. 2(b). The estimates are accurate 

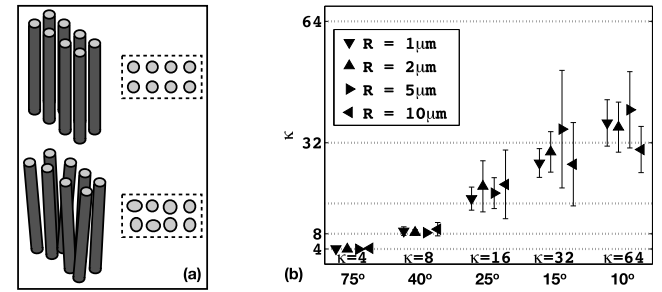

Fig. 2. (a) The schematic illustration of the overestimation of axon diameters due to orientation dispersion. (b) The estimated $\kappa$ using the Watson model from the synthetic experiments. The dotted lines indicate the true $\kappa$ values. The numerics underneath the $\mathrm{x}$-axis indcate the polar angle value containing $95 \%$ of orientation variation in the corresponding Watson distribution.
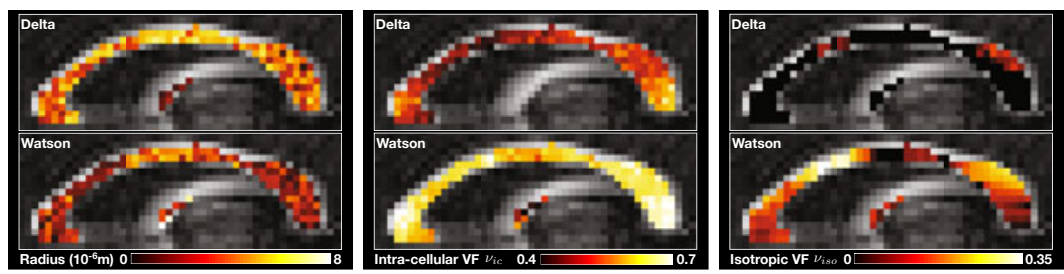

Fig. 3. The parameter estimates for the midsagittal slice of CC using the Delta and Watson models

for smaller $\kappa$ values but become increasingly biased downward for higher $\kappa$. The highest values, 32 and 64, are more difficult to distinguish.

In Vivo Human Brain Data Experiments. Here we fit each model to real brain data. To avoid regions with fiber crossing, we compute the linearity and planarity [14] maps of the best-fit diffusion tensor and select only the voxels with high linearity, greater than 0.6, and low planarity, less than 0.2 .

Fig. 3 shows the estimated parameters from both models in the midsagittal slice of CC. The corresponding $\kappa$ estimates are shown in Fig. 4 (bottom row \& middle column). The results agree strongly with the trends observed in the synthetic experiments, i.e., for $\kappa$ lower than 32 , the Delta model overestimates $R$ and underestimates $\nu_{i c}$ and $\nu_{i s o}$. They are also much more consistent with histology [15] than results from the Delta model, i.e., axons have smaller radii, are more densely packed (higher $\nu_{i c}$ ) and coherent (higher $\kappa$ ) in the genu and splenium of CC than its midbody. Fig. 4] shows additional results of the estimates of $R$ from both models and the estimates of $\kappa$ from the Watson model for two lateral slices in addition to the full midsagittal slice. Besides confirming the trend observed above, it shows that the estimates of $\kappa$ in the genu and the splenium of $\mathrm{CC}$, as expected, are the highest for the whole brain. 


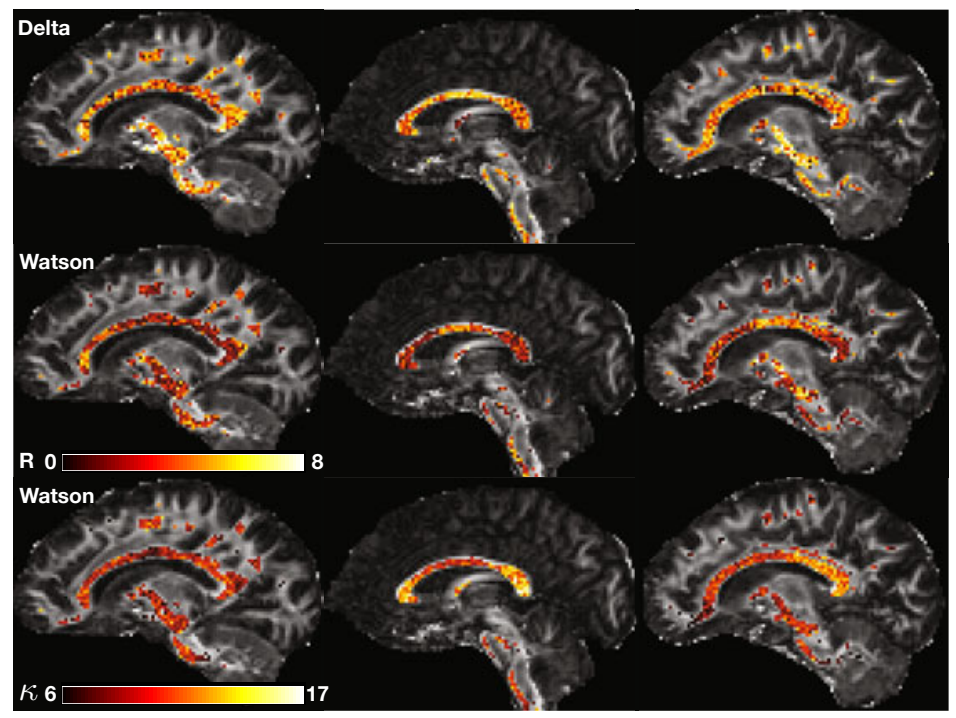

Fig. 4. The estimates of $R$ for 3 sagittal slices using the Delta (top) and Watson (middle) models, with the corresponding $\kappa$ estimates (bottom) from the Watson model

\section{Discussion}

In this paper, we proposed a new white matter tissue model for the direct estimation of axon diameters and other microstructural features using diffusion MRI. The proposed model advances the state-of-the-art by explicitly accounting for the presence of orientation dispersion in axon bundles to mitigate the overestimation bias in estimating axon diameters. Synthetic experiments demonstrate that, in the presence of orientation dispersion, models assuming a single orientation not only overestimates axon diameters but also underestimates intra-cellular volume fractions and biases isotropic volume fractions. In contrast, the proposed model can both estimate these key tissue parameters more accurately without systematic bias and provide estimates to the extent of orientation dispersion. Results from in vivo human data experiments agree strongly with the findings from simulation, which suggests the orientation dispersion must be taken into account even for the most coherent structures like CC and CST.

One possible limitation of the present approach is its use of the Watson distribution to model the underlying orientation dispersion in brain data. For voxels with significant spanning or bending axon bundles, the cylindrical symmetry of the Watson distribution may prove overly simplistic and introduce potential bias in estimating microstructural parameters. Future work will explore the use of more complex orientation distributions, such as the Bingham distribution [1], to model such effects. 
Acknowledgment. We thank Penny Hubbard and Geoffrey Parker from University of Manchester for providing the in-vivo human dataset. The future and emerging technologies (FET) program of the EU FP7 framework fund the CONNECT consortium (www.brain-connect.eu), which supports this work. EPSRC fund DCA under grant EP/E007748.

\section{References}

1. Basser, P.J., Mattiello, J., Bihan, D.L.: MR diffusion tensor specstroscopy and imaging. Biophys. J. 66 (1994)

2. Pierpaoli, C., Jezzard, P., Basser, P.J., Barnett, A., Chiro, G.D.: Diffusion tensor MR imaging of the human brain. Radiology 201 (1996)

3. Stanisz, G.J., Szafer, A., Wright, G.A., Henkelman, M.: An analytical model of restricted diffusion in bovine optic nerve. MRM 37, 103-111 (1997)

4. Assaf, Y., Freidlin, R.Z., Rhode, G.K., Basser, P.J.: New modeling and experimental framework to characterize hindered and restricted water diffusion in brain white matter. MRM 52, 965-978 (2004)

5. Assaf, Y., Blumenfeld-Katzir, T., Yovel, Y., Basser, P.J.: AxCaliber: a method for measuring axon diameter distribution from diffusion MRI. MRM 59, 1347-1354 (2008)

6. Alexander, D.C.: A general framework for experiment design in diffusion MRI and its application in measuring direct tissue-microstructure features. MRM 60, 439 448 (2008)

7. Barazany, D., Basser, P.J., Assaf, Y.: In-vivo measurement of the axon diameter distribution in the corpus callosum of a rat brain. Brain 132, 1210-1220 (2009)

8. Alexander, D.C., Hubbard, P.L., Hall, M.G., Moore, E.A., Ptito, M., Parker, G.J.M., Dyrby, T.B.: Orientationally invariant indices of axon diameter and density from diffusion MRI. NeuroImage (2010), doi:10.1016/j.neuroimage.2010.05.043

9. Jespersen, S.N., Kroenke, C.D., Ostergaard, L., Ackerman, J.J.H., Yablonskiy, D.A.: Modeling dendrite density from magnetic resonance diffusion measurements. NeuroImage 34, 1473-1486 (2007)

10. Murday, J.S., Cotts, R.M.: Self-diffusion coefficient of liquid lithium. J. Chem. Phys 48, 4938-4945 (1968)

11. Mardia, K.V., Jupp, P.E.: Directional statistics. Wiley series in probability and statistics. John Wiley \& Sons, Ltd., Chichester (1990)

12. Abramowitz, M., Stegun, I.A.: Handbook of mathematical functions with formulas, graphs, and mathematical tables, 9th edn. Dover, New York (1972)

13. Szafer, A., Zhong, J.H., Gore, J.C.: Theoretical model for water diffusion in tissues. MRM 33, 697-712 (1995)

14. Westin, C.F., Maier, S.E., Mamata, H., Nabavi, A., Jolesz, F.A., Kikinis, R.: Processing and visualization for diffusion tensor MRI. MIA 6, 93-108 (2002)

15. Aboitiz, F., Scheibel, A.B., Fisher, R.S., Zaidel, E.: Fiber composition of the human corpus callosum. Brain research 598, 143-153 (1992) 